\title{
APRENDIZAJE ORGANIZACIONAL Y SU VINCULACIÓN CON LA COMUNICACIÓN
}

\author{
Iván Rueda Fierro* \\ Grace Natalie Tamayo** \\ Byron Acosta Andino*** \\ Fabián Cueva Brito**** \\ Paul Idrobo Dávalos****
}

\begin{abstract}
Resumen. Esta investigación tiene como objetivo analizar el aprendizaje organizacional y su vinculación con la comunicación, mediante una revisión de sus fundamentos teóricos y la identificación de las variables más representativas de estos aportes en el contexto organizacional. Se concluye que la comunicación es reconocida como un factor crítico en los procesos de conocimiento y la interacción de los grupos, y se ha convertido en la principal fuente de ventaja competitiva para las organizaciones, cuya creación dependerá de las condiciones organizacionales presentes. Cuando una organización tiene una cultura organizacional basada en el aprendizaje, el conocimiento de los trabajadores se integra y genera soluciones para los problemas. Para este ciclo se requiere que los directivos de las organizaciones asuman con liderazgo el proceso de gestión del conocimiento, conjuntamente con el compromiso del personal y alineen la estrategia con la cultura organizacional. En consecuencia, bajo estas condiciones se permitirá la generación, difusión y transferencia del conocimiento. Es importante para las instituciones conocer y controlar la relación entre el estilo de comunicación usado y los procesos de conocimiento.
\end{abstract}

Palabras Clave: Organizaciones; Comunicación organizacional; Tipologías de aprendizaje organizacional.

\footnotetext{
* Pontificia Universidad Católica del Ecuador (PUCE), Ecuador.

Contacto: iarueda@puce.edu.ec

** Instituto de Altos Estudios Nacionales (IAEN), Ecuador.

Contacto: gracen.tamayo@gmail.com

*** Escuela Politécnica Nacional (EPN), Ecuador.

Contacto: byron@hotmail.com.br

**** Pontificia Universidad Católica del Ecuador (PUCE), Ecuador.

Contacto: efcuevab@puce.edu.ec

***** Pontificia Universidad Católica del Ecuador (PUCE), Ecuador.

Contacto: pmidrobo@puce.edu.ec
} 


\title{
ORGANIZATIONAL LEARNING AND ITS LINK WITH COMMUNICATION
}

\begin{abstract}
The objective of this research is to analyze organizational learning and its relationship with communication, through a review of its theoretical foundations and the identification of the most representative variables of these contributions in the organizational context. It is concluded that communication is recognized as a critical factor in the processes of knowledge and interaction of groups and has become the main source of competitive advantage for organizations, whose creation will depend on the organizational conditions present. When an organization has an organizational culture based on learning, the knowledge of the staff is integrated and generates solutions for the problems. For this cycle it is required that the managers of the organizations assume with leadership the process of knowledge management, together with the commitment of the personnel and align the strategy with the organizational culture. Consequently, under these conditions the generation, diffusion and transfer of knowledge will be allowed. It is important for institutions to know and control the relationship between the communication style used and the knowledge processes.
\end{abstract}

Keywords: Organizations; Organizational communication; Typologies of organizational learning.

Original recibido el 06/03/2018

Aceptado para su publicación el 14/11/2019 


\section{Introducción}

El término, aprendizaje organizacional fue usado por primera vez por March y Simón en 1958 y debe su difusión a Senge, quien lo abordó en su libro la Quinta Disciplina de 1990 (Cortina, Fernando, y Rojas, 2011). A partir de la década de los ochenta se produce un creciente interés en el estudio del aprendizaje organizacional.

La teoría relacionada con la creación del conocimiento organizacional fue desarrollada por Nonaka y Takeuchi (1995) quienes la definen como la capacidad que tiene una organización para generar conocimiento, difundirlo en su conjunto y materializarlo en procesos, productos y servicios (Fernández, 2007).

De esta manera, las organizaciones que aprenden son posibles porque en el fondo todos somos aprendices. El aprendizaje es algo innato en las personas y las personas son las que conforman las organizaciones y hacen que éstas también aprendan (Senge, 1990).

Por lo tanto, el aprendizaje organizacional es un proceso a través del cual las diferentes entidades, de cualquier tamaño y orientación, obtienen y generan conocimiento mediante sus empleados, con el objetivo de convertirlo en conocimiento institucional (Cortina, Fernando, y Rojas, 2011). Para que este conocimiento pueda ser aprovechado se necesitan procesos que permitan comunicar, difundir, compartir y transmitir la información. El reto de la comunicación consiste en asumir su función estratégica y formativa en la organización que aprende, generando estrategias adecuadas de comunicación para que el conocimiento organizativo se mantenga disponible y accesible, agilizando los procesos de toma de decisiones y mejorando la calidad (Martín, 2007). Con base en este contexto el objetivo de esta revisión bibliográfica es analizar el aprendizaje organizacional y su vinculación con la comunicación.

\section{Aprendizaje organizacional y sus tipologías}

Nonaka y Tekeuchi desarrollan una teoría sobre la creación de conocimiento en las organizaciones, sustentada en sus investigaciones realizadas en empresas japonesas y en la posibilidad de complementar su enfoque con la práctica de la gestión en las empresas occidentales. Su teoría parte del principio de que el conocimiento puede ser tácito o explícito. La diferencia entre ambos tipos indica cuatro patrones o formas para crear conocimiento en cualquier entidad (Fernández, 2007; López, Cabrales, y Schamal, 2005; Nonaka y Takeuchi, 2000):

1) De tácito a tácito: se produce a través de la socialización. Implica participar de los conocimientos tácitos de cada persona. Este conocimiento se desarrolla al compartir modelos mentales, aptitudes y habilidades y al involucrar la aceptación de creencias, sentimientos y emociones de otros, es muy difícil de lograr sin el contacto cara a cara. Además, debido a que este conocimiento no se vuelve explícito, la organización no lo puede aprovechar fácilmente.

2) De explícito a explícito: se da por medio de la asociación o combinación que consiste en transformar el conocimiento explícito en formas más complejas de este mismo tipo de conocimiento.

3) De tácito a explícito: se da por la exteriorización, que significa que el conocimiento 
tácito es articulado en conceptos explícitos de manera que el conocimiento tácito pueda entenderse por los demás. Este proceso se desarrolla mediante el diálogo y la reflexión conjunta, usando diferentes métodos de razonamiento como la inducción, deducción y abducción.

4) De explícito a tácito: se crea por la interiorización, la cual consiste en la conversión del recién creado conocimiento explícito, en tácito, desarrollado por el aprendizaje experimental. Esto se produce cuando el nuevo conocimiento explícito es compartido en la organización, otros empleados lo asimilan y lo utilizan para ampliar, reformular o extender su propio conocimiento. Este proceso permite que el nuevo conocimiento se consolide en los elementos cognitivos y técnicos que cada persona posee.

Alcover y Gil (2002), reconocen tres tipos de aprendizaje: aprendizaje de bucle sencillo o correctivo, aprendizaje de doble bucle o adaptativo y aprendizaje de triple bucle o como solución de problemas. El primer tipo de aprendizaje se basa en solucionar las desviaciones producidas mediante la correspondiente corrección o ajuste de los procedimientos establecidos. El segundo tipo, ocurre cuando los sistemas de interpretación de la organización son redefinidos o cambiados para adaptarse a las demandas del ambiente. Por último, el tercer tipo de aprendizaje se genera a través de la reflexión colectiva sobre las creencias y fundamentos que se sostienen en la organización y se retan los modelos mentales colectivos de la cultura. En este último, se busca desarrollar la capacidad de un sistema para autoguiarse con referencia a un conjunto de normas y valores, cuestionándolas como guías apropiadas de comportamiento

De esta forma, se puede visualizar que el aprendizaje organizacional es un proceso de adquisición y creación del conocimiento institucional. Este proceso, según Castañeda (2015) se da en tres niveles: individual, grupal y organizacional. Adicionalmente, se requieren cuatro condiciones para que este proceso se desarrolle en las organizaciones. La primera condición es el fomento de una cultura de aprendizaje caracterizada por la conexión entre compartir el conocimiento para solucionar los problemas y una activa participación de los empleados en la solución de los mismos. La segunda, es la formación de los trabajadores. La tercera, es la claridad estratégica, implica que la misión, visión, objetivos y estrategias sean conocidos y compartidos por los miembros de la organización. La cuarta y última condición es el soporte organizacional, que implica que la empresa proporcione todos los recursos físicos y tecnológicos necesarios para que el conocimiento se divulgue y se comparta.

Cuando un trabajador adquiere conocimiento y desarrolla sus habilidades, es más probable que incremente su desempeño y, por ende, contribuya al logro de los objetivos organizacionales. De esta forma, sus aprendizajes están alineados con el conocimiento clave para lograr los objetivos estratégicos y al final produce que la organización aprenda (Castañeda, 2015).

Por lo tanto, la capacidad de aprendizaje se define como el potencial dinámico de creación, asimilación, difusión y utilización del conocimiento que se da por medio de la formación y evolución de los stocks de conocimiento (Oviedo-García et al., 2014). Para estos autores, los stocks de conocimiento son de tres tipos: conocimientos 
individuales que se dan por aprendizaje individual y forman parte del capital humano de la organización; conocimientos de grupo que se dan por el entendimiento compartido entre las personas; y, por último, conocimientos organizativos que suponen traspasar los dos tipos de conocimiento anteriores a sistemas, estructuras, procedimientos, rutinas y experiencias desarrolladas por la organización (OviedoGarcía et al., 2014).

A manera de resumen se exponen los principales aportes que se han dado referente al aprendizaje organizacional (Tabla 1).

\section{Tabla 1. Aportes en aprendizaje organizacional}

\begin{tabular}{|c|c|c|c|}
\hline Año & Autores & Aporte & $\begin{array}{l}\text { Variable más } \\
\text { representativa }\end{array}$ \\
\hline 1993 & Dogson & $\begin{array}{l}\text { A través del aprendizaje organizacional se mejoran } \\
\text { las habilidades de fuerza de trabajo. }\end{array}$ & $\begin{array}{l}\text { Trabajo en equipo, } \\
\text { capacidades } \\
\text { individuales. }\end{array}$ \\
\hline 1994 & Garvin & $\begin{array}{l}\text { La organización es capaz de crear, adquirir, } \\
\text { transferir conocimiento. }\end{array}$ & $\begin{array}{l}\text { Conocimientos, } \\
\text { competitividad. }\end{array}$ \\
\hline 1996 & Marquardt & $\begin{array}{l}\text { Organización que aprende es una organización que } \\
\text { aprende colectivamente y se transforma o cambia } \\
\text { continuamente. }\end{array}$ & $\begin{array}{l}\text { Trabajo en equipo, } \\
\text { cambios. }\end{array}$ \\
\hline 1998 & De Geus & $\begin{array}{l}\text { La única ventaja competitiva que tiene una } \\
\text { organización es la de adquirir conocimiento. }\end{array}$ & $\begin{array}{l}\text { Conocimiento, } \\
\text { competitividad }\end{array}$ \\
\hline 1999 & $\begin{array}{l}\text { Argyris y } \\
\text { Schon }\end{array}$ & $\begin{array}{l}\text { El aprendizaje organizacional es un proceso } \\
\text { antirutinario. }\end{array}$ & Proceso \\
\hline 1999 & Zack & $\begin{array}{l}\text { Aprendizaje Organizacional es la habilidad para } \\
\text { explotar el conocimiento mejor que la competencia. }\end{array}$ & $\begin{array}{l}\text { Competi } \\
\text { conocim }\end{array}$ \\
\hline 1999 & Drucker & $\begin{array}{l}\text { A través del aprendizaje la organización puede } \\
\text { adaptarse a los cambios del entorno }\end{array}$ & $\begin{array}{l}\text { Cambios del } \\
\text { entorno }\end{array}$ \\
\hline 1999 & $\begin{array}{l}\text { Nonaka y } \\
\text { Takeuchi }\end{array}$ & $\begin{array}{l}\text { Para convertir el conocimiento individual en } \\
\text { organizacional deben existir diálogo, discusión, } \\
\text { observación, imitación, práctica y experimentación }\end{array}$ & $\begin{array}{l}\text { Conocimiento, } \\
\text { comportamiento } \\
\text { organizacional }\end{array}$ \\
\hline 1999 & Choo & $\begin{array}{l}\text { Las organizaciones crean nuevo conocimiento a } \\
\text { partir de las experiencias de sus empleados }\end{array}$ & $\begin{array}{l}\text { Capacidades } \\
\text { individuales }\end{array}$ \\
\hline 1999 & Nonaka & $\begin{array}{l}\text { La creación del conocimiento organizacional } \\
\text { comienza con el esfuerzo individual }\end{array}$ & $\begin{array}{l}\text { Capacidades } \\
\text { individuales }\end{array}$ \\
\hline 2000 & Aramburu & $\begin{array}{l}\text { El aprendizaje organizacional está asociado tanto } \\
\text { al cambio del comportamiento organizativo como } \\
\text { la creación de una base de conocimiento que la } \\
\text { soporte }\end{array}$ & Trabajo en equipo \\
\hline 2001 & $\begin{array}{l}\text { Ciborra y } \\
\text { Andreu }\end{array}$ & $\begin{array}{l}\text { El aprendizaje organizacional es un sistema de } \\
\text { recurso y capacidades }\end{array}$ & $\begin{array}{l}\text { Capacidades } \\
\text { individuales }\end{array}$ \\
\hline 2002 & Camison & $\begin{array}{l}\text { El aprendizaje organizacional permite mantener } \\
\text { ventaja competitiva }\end{array}$ & $\begin{array}{l}\text { Ventaja } \\
\text { competitiva }\end{array}$ \\
\hline 2003 & López & $\begin{array}{l}\text { El aprendizaje en equipo es una instancia que } \\
\text { permite encausar los nuevos conocimientos } \\
\text { y habilidades adquiridas por cada uno de los } \\
\text { miembros. }\end{array}$ & $\begin{array}{l}\text { Trabajo en equipo, } \\
\text { conocimientos }\end{array}$ \\
\hline
\end{tabular}

Fuente: Cortina et al. (2011).

En general, más allá de los términos utilizados, lo que se distingue en los distintos aportes, es la consideración del aprendizaje como una respuesta condicionada, así como la concepción de la organización como un sistema racional adaptativo que 
aprende sobretodo de la experiencia y la consideración del aprendizaje como el resultado de un proceso de maduración y reflexión.

\section{La relación entre aprendizaje organizacional y comunicación}

Núñez Paula (2004) afirma que la comunicación es un proceso en el que cada persona actúa simultáneamente como fuente o emisor de mensajes, que traslada ideas, actitudes, valores, sentimientos, y como receptor o destino de mensajes de otros que portan los mismos elementos. Para Tobio (1997), existen tres estados de conciencia de la importancia de la comunicación organizacional en las organizaciones: en el primero la comunicación es vista como un proceso espontáneo y no se percibe la necesidad de considerarle como objeto de gestión y ocurre básicamente mediante canales informales. En el segundo estado intermedio, la organización ve la necesidad de sistematizar su comunicación interna y se establecen mecanismos para que la información fluya por ella. En el tercer estado la comunicación se entiende como un medio que debe contribuir activamente a la transformación de la organización y adquiere un carácter estratégico para gestionar el cambio.

Es importante definir la información interna y la comunicación con el entorno. Para Núñez Paula (2004), la primera conforma la cultura e identidad de la organización y condiciona el contenido y la forma de comunicación con el entorno, mientras que esta última constituye el punto de partida y destino de la planificación estratégica, el posicionamiento, la toma de decisiones, la gestión de procesos y el mejoramiento continuo para el aprendizaje organizacional.

Para Martín (2007) el uso de la información y el conocimiento pasa por tres fases: en la primera, la información y la tecnología se organizan internamente sin tener relación una con otra. En la segunda, ante el crecimiento del volumen de la información surge la gestión de la información que incluye la infraestructura tecnológica necesaria para acceder rápidamente a la información. La tercera fase se produce cuando la organización genera y comparte conocimiento y está en capacidad de realizar cambios e innovar a partir de ese aprendizaje.

Al relacionar el aprendizaje con la comunicación, se verifica que todas las organizaciones aprenden, ya que aprender es adaptarse a medida que el mundo cambia a su alrededor, con la diferencia de que unas cambian de forma más eficiente y rápida que otras, es decir, son mejores aprendices (Senge et al., 2000). La utilización de conocimiento, cuando es visto desde la teoría de la comunicación, se entiende mejor cuando se concentran en las barreras que impiden la transferencia eficiente de la información. En cualquier sistema de comunicación humana se desarrollan diversas fuerzas que inhiben la transferencia efectiva de significados (Duncan, 1973). Las barreras más importantes con que se encuentran las organizaciones para convertirse en organizaciones que aprenden, a través de la gestión del conocimiento, tiene que ver con la dificultad para la evolución de la cultura interna, dificultad para identificar información relevante, existencia de brechas de competencias individuales y grupales, falta de espacios, canales y herramientas adecuadas para almacenar, distribuir y permitir el acceso a la comunicación y dificultad para reconocer el aprendizaje organizacional como estrategia (Martín 2007).

Para eliminar estas barreras, es clave el papel de la comunicación como formadora, 
transmisora y difusora de valores culturales. Para Martín (2007), la comunicación apoya y logra la evolución cultural necesaria en la organización para generar una cultura de innovación basada en conocimientos, ya que la comunicación por medio de la interacción entre personas, hace efectivos los procesos de cuestionamiento, validación desarrollo de presupuestos, siendo todos éstos condicionantes de la gestión del conocimiento. El uso de las tecnologías de la información y la comunicación se ha convertido en una herramienta estratégica que ha dado un cambio total a las diferentes tácticas que podrían implementarse para mejorar los procesos dentro de las organizaciones (Manzano, Peñaranda y Páez, 2019).

En consecuencia, la generación de conocimiento no es simplemente procesar información objetiva, sino aprovechar las intuiciones tácitas, y a menudo subjetivas, de los empleados y ponerlas a disposición de la organización para que las pruebe y las utilice. Como los sostienen Nonaka y Takeuchi (2000), la clave está en el compromiso y la identificación que tienen los empleados con su organización.

El conocimiento, por lo tanto, es el recurso estratégico más importante para las organizaciones y su capacidad para gestionarlo se constituye en un aspecto relevante para sostener una ventaja competitiva (Oviedo-García et al., 2014). La gestión del conocimiento debe integrar la gestión de información, mediante una comunicación que involucre a la organización como a un todo, considera la compleja interacción con sus miembros y grupos (Núñez Paula, 2004).

No solo es importante lograr conocimiento y aprendizaje individual, sino que además es importante que las entidades logren generar, fomentar y gestionar el conocimiento de forma sistemática y metódica, para que éste se incorpore al aprendizaje de la organización (Encinas Orozco, 2014). Desarrollar las capacidades de aprendizaje en las personas y sus equipos de trabajo conduce a impulsar el crecimiento y éxito en las organizaciones (Senge et al., 2000). Generar conocimiento significa crear nuevamente a la empresa y a todos sus miembros en un proceso ininterrumpido de autorrenovación personal y organizacional (Nonaka y Takeuchi, 2000).

De los cuatro modos de conversión del conocimiento propuestos por Nonaka y Takeuchi, la exteriorización (de tácito a explícito) es la clave para la creación de conocimiento, porque genera nuevos conceptos que emanan del conocimiento implícito (Fernández, 2007). Para crear este conocimiento, las organizaciones deben vincular ideas y cosas contradictorias mediante metáforas, para después resolver estas contradicciones mediante analogías y finalmente precisar los conceptos creados en un modelo para poner el conocimiento a disposición del resto de la organización (Nonaka y Takeuchi, 2000). Para esto se requiere motivación específica para enseñar y comunicar.

Las razones anteriormente expuestas permiten conceptualizar al aprendizaje organizacional como proceso dinámico, que debe generarse en el tiempo y traspasar sus fronteras. Esto podría generar tensión entre la asimilación del nuevo conocimiento y el uso del aprendido. Dicha tensión tiende a aumentar en el aprendizaje institucionalizado, porque el mismo tiende a obstaculizar la asimilación del nuevo conocimiento (López et al., 2005).

Este conocimiento que lleva a la acción debe comunicarse por todos los medios 
posibles para facilitar su acceso a los miembros de cada entidad, de manera tal que lo utilicen en la resolución de sus propios problemas, y a su vez, generen retroalimentación al sistema con conocimiento nuevo que sea continuamente revisado, utilizado, validado y mejorado (Oviedo-García et al., 2014).

Esta información procesada, producto del acto comunicativo de transformación del conocimiento, no necesariamente implica un proceso de comunicación, ya que para que éste se produzca se requiere que la información fluya, al menos en sentido directo e inverso, entre los que se comunican (Núñez Paula, 2004).

Es importante, además del desarrollo de los procesos de comunicación, la generación de condiciones facilitadoras de la gestión del conocimiento, tales como: el liderazgo de los directivos, junto con la colaboración y, sobre todo, el compromiso de todos los miembros para generar el efecto positivo del aprendizaje organizacional (AcostaPrado y Eiko Nakata, 2012). Como lo mencionan Alavi y Leidner (1999), los beneficios de la gestión del conocimiento serán aprovechados por las organizaciones que no sean solamente aptas desde el aspecto tecnológico; sino que además inviertan a largo plazo para alinear los elementos culturales, de gestión y de organización con la gestión del conocimiento.

Para crear esta estructura que permita generar, utilizar y comunicar el conocimiento organizacional, se requiere el establecimiento de un lenguaje común en la comunicación para articular concepciones comunes, tanto cognitivas como afectivas. Adicionalmente, para garantizar la comprensión y uso adecuado de la información, se requiere de formas específicas de comunicación basadas en las necesidades de formación e información de las personas y de la organización como un todo (Núñez Paula, 2004). De esta forma, la comunicación debe crear un mayor sentido de conexión entre todos los niveles. Los sistemas de decisión se deben usar para facilitar el acceso a la información, su difusión e interpretación compartida entre todos los miembros de la organización (Barker y Camarata, 1998).

Las organizaciones exitosas serán aquellas que articulen la estrategia y las necesidades de sus colaboradores; así como sus necesidades de conocer, compartir y aprender para ejecutar dicha estrategia. Esta articulación debe guiar el despliegue de los recursos organizacionales y tecnológicos, así como movilizar las capacidades de sus empleados para aprovechar al máximo el conocimiento, lo que incrementa las habilidades organizacionales para competir y reaccionar ante los cambios del entorno (Cortina et al., 2011). Consecuentemente, la gestión de la información es condición necesaria para la gestión del conocimiento ya que implica la socialización del conocimiento tácito de los miembros de la organización, su interiorización, el uso compartido de los conocimientos y la generación y registro del conocimiento organizacional (Núñez Paula, 2004).

Para esto es necesario considerar a los flujos de conocimiento, tanto explotadores como exploradores, como componentes de la capacidad de aprendizaje de las organizaciones que ejercen mayor impacto en el desempeño organizacional (OviedoGarcía et al., 2014). Por lo tanto, es posible que las organizaciones que enfoquen sus estrategias al desarrollo del aprendizaje organizacional consigan mejores resultados, implicando el desarrollo de instrumentos y estructuras que permitan 
recoger el conocimiento adquirido y transmitirlo. Como lo sostiene Castañeda (2015), el proceso de creación y adquisición de conocimiento no es un proceso espontáneo que se da de forma natural, sino que requiere de condiciones organizacionales que lo promuevan.

Así, las organizaciones que tendrán mayor relevancia en el futuro serán las que descubran como aprovechar el entusiasmo y la capacidad de aprendizaje y comunicación de todos sus empleados, sin importar el nivel que ocupen en la organización (Senge, 1990).

Marulanda, Giraldo, y López (2013) realizaron una investigación para establecer el estado de la gestión del conocimiento referente a las tecnologías de información y comunicación en la región del eje cafetero de Colombia. Para tal efecto, aplicaron una encuesta a 22 organizaciones y empresas, para evaluar dos constructos: 1) competencias de gestión de la información y la documentación, gestión de la comunicación diseño de herramientas digitales, gestión de la innovación y el cambio y gestión del aprendizaje organizacional, y 2) prácticas de identificación, generación, retención, difusión y aplicación de conocimiento. Como conclusión se obtuvo que se requiere mayor consolidación en las prácticas de gestión del conocimiento, para lo que se propone que las empresas incorporen la gestión del conocimiento mediante el manejo direccionado de los procesos de conocimiento y formen al capital humano en gestión de la información, en gestión de la innovación y en gestión de la comunicación.

Michailova y Sidorova (2011) proponen un modelo conceptual centrado en la idea que el trabajo en grupo sirve como herramienta para el aprendizaje organizacional, influenciado por la comunicación y los procesos de compartición de conocimientos. Este modelo sugiere que el intercambio de conocimientos en el grupo está mediado preferentemente por las formas de comunicación y luego por las variaciones culturales de los miembros del grupo (Figura 1).

Figura 1. Grupo de trabajo, intercambio de conocimientos y aprendizaje organizacional

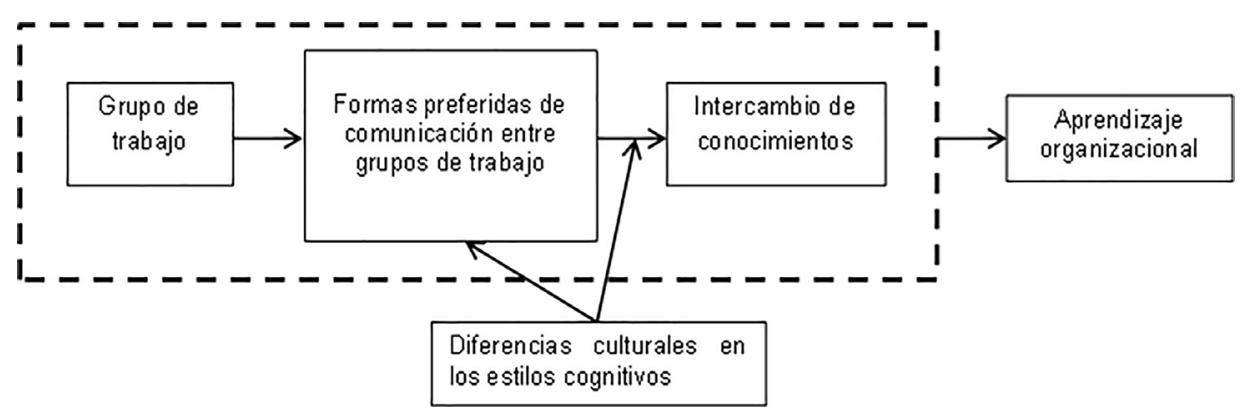

Fuente: Michailova y Sidorova, (2011).

Este modelo conceptual se basa en la idea de que la comunicación juega un papel de suma importancia en el intercambio de conocimientos y el aprendizaje organizacional. La comunicación es reconocida como un factor crítico en los procesos de conocimiento y la interacción de los grupos; por lo tanto, la eficacia de los mismos 
se basa en el estilo de comunicación usado; es decir, que el tipo de comunicación tiene un potencial diferenciador para facilitar el intercambio de conocimientos en diferentes contextos culturales.

Martínez Crespo y Giraldo Marín (2012), analizaron la dificultad que tienen las organizaciones para articular su gestión a las tecnologías de información y comunicación en la implementación de proyectos de gestión del conocimiento. Los resultados fueron que las tecnologías de información y comunicación constituyen apenas un $10 \%$ de todo el proceso de gestión del conocimiento en una empresa, es decir, su existencia no garantiza el logro de los resultados que se esperan obtener. Además, el componente humano es el factor más determinante para el éxito de los proyectos de gestión del conocimiento, pero deben ser ligados a la estrategia con procesos bien establecidos y con estructuras flexibles que permitan, sobre todo, la libre movilidad de la información y el conocimiento. La adaptación de la estructura, la cultura y la estrategia a los cambios propuestos por los proyectos de gestión del conocimiento en la empresa, sumada al soporte de las tecnologías de la información para que fluya la comunicación entre los miembros, garantiza un mejor desempeño de la organización (Martínez Crespo y Giraldo Marín, 2012).

Dimovski y Škerlavaj (2004) desarrollaron un modelo para determinar la relación entre el aprendizaje organizacional y las tecnologías de información y comunicación (Tabla 2), que fue aplicado en 220 empresas de Eslovenia con más de 100 trabajadores. Como resultado se encontró que existe una correlación moderada y positiva entre el aprendizaje organizacional y las tecnologías de información y comunicación lo que determina que un mejor aprendizaje organizacional contribuye a un mejor uso, despliegue y gestión de tecnologías modernas de información y comunicación. Al mismo tiempo las TIC [tecnologías de la información y la comunicación] pueden ser vistas como facilitadores del aprendizaje organizacional.

\section{Tabla 2. Especificación de variables}

\begin{tabular}{|c|c|}
\hline Variables & Variables de medición \\
\hline Aprendizaje Organizacional & $\begin{array}{c}\text { Adquisición de información } \\
\text { Interpretación de información } \\
\text { Cambios cognitivos y conductuales }\end{array}$ \\
\hline Tecnologías de información y comunicación & $\begin{array}{c}\text { Hardware } \\
\text { Software }\end{array}$ \\
\hline
\end{tabular}

Fuente: Dimovski y Škerlavaj (2004).

Estudios más recientes confirman la relación positiva entre las tecnologías de información y comunicación y el aprendizaje organizacional. En un estudio realizado por Manzano, Peñaranda, \& Páez (2019) en las micro, pequeñas y medianas empresas de la ciudad de Ocaña en Colombia determinó que existe un impacto positivo y significativo entre las tecnologías de información y comunicación y el aprendizaje organizacional lo que mejora la competitividad. Las herramientas TIC sugeridas para mejorar la competividad en las MiPyMEs [micro, pequeñas y medianas empresas] son: intranet, correo electrónico, videoconferencias, redes sociales, motores de búsqueda, gestión de documentos, comercio electrónico, bases de datos, realidad virtual, inteligencia artificial y aplicaciones de soporte para 
cada tipo de organización.

Dos estudios similares aplicados en la industria de alta tecnología en China realizados por Huang y Yao (2017) y Duan (2017) obtuvieron los siguientes resultados: la influencia del aprendizaje organizacional en la comunicación organizacional es positivamente significativo, la influencia de la comunicación organizacional en la creatividad organizacional es positiva y la influencia de del aprendizaje organizacional en la creatividad organizacional es positivo.

Por lo anteriormente expuesto, se puede apreciar que en el entorno empresarial actual es muy importante realizar esfuerzos para lograr el aprendizaje organizacional, para lo cual se requiere la planificación estratégica de los sistemas de información y que los directivos generen una cultura organizativa de confianza para que la información sea compartida (Dimovski y Škerlavaj, 2004).

\section{Conclusiones}

El objetivo de esta revisión fue analizar el aprendizaje organizacional y su vinculación con la comunicación. Se observa que el conocimiento se ha convertido en la principal fuente de ventaja competitiva para las organizaciones que se han dedicado desde sus comienzos a la generación y trasmisión del conocimiento. La adquisición y creación de conocimiento depende de las condiciones organizacionales presentes. Cuando una organización tiene desarrollada una cultura organizacional basada en el aprendizaje, el conocimiento de los trabajadores se integra y genera soluciones para los problemas que se presenten.

Además, se requiere que los directivos de las organizaciones asuman con liderazgo el proceso de gestión del conocimiento, comprometan al personal y alineen la estrategia con la cultura organizacional, de manera que ésta permita la generación, difusión y transferencia del conocimiento. El proceso de creación y gestión del conocimiento no se da de forma natural en las organizaciones, sino que debe promoverse. Es importante, por lo tanto, que se establezcan procesos sistemáticos de comunicación para aprovechar el conocimiento generado, que permitan difundirlo, implementarlo y mejorarlo.

Cualquier organización que quiera transformarse en una organización que aprende necesita la intervención de la comunicación para actuar como medio de formación, transmisión, difusión y distribución de los nuevos valores culturales que determinen el comportamiento nuevo de las personas. Las tecnologías de la información mejoran la comunicación y, por lo tanto, optimizan la gestión del conocimiento, aunque no son el único factor que incide en el éxito para aprovechar el conocimiento generado. Se requieren realizar más estudios relacionados con el aprendizaje y la comunicación, sean éstos longitudinales o incluyendo nuevos sectores empresariales.

\section{Referencias bibliográficas}

Acosta-Prado, J. C. y Eiko Nakata, L. (2012). Condiciones de la gestión del conocimiento que favorecen el aprendizaje organizacional: un estudio comparativo. Revista Sotavento, 20, pp. 22-38. https://doi.org/10.2139/ 
Alavi, M. y Leidner, D. (1999). Knowledge management systems: issues, challenges, and benefits. Communications of the AIS, 1(1), pp. 1-38.

Alcover, C. M. y Gil, F. (2002). Crear conocimiento colectivamente: aprendizaje organizacional y grupal. Revista de Psicología del Trabajo y de las organizaciones, 18(2-3), pp. 259-301.

Barker, R. T. y Camarata, M. R. (1998). The role of communication in creating and maintaining a learning organization: Preconditions, indicators, and disciplines. The Journal of Business Communication (1973), 35(4), pp. 443-467.

Castañeda, D. I. (2015). Condiciones para el aprendizaje organizacional. Estudios Gerenciales, 31(134), pp.62-67.https://doi.org/10.1016/j.estger.2014.09.003

Cortina, R., Fernando, D. y Rojas, S. (2011). Perspectivas del aprendizaje organizacional como catalizador de escenarios competitivos. Revista Ciencias Estratégicas, 19(26), 247-266.

Dimovski, V. y Škerlavaj, M. (2004). Organizational learning and informationcommunication technologies: a promising link. Zbornik radova Ekonomskog fakulteta u Rijeci: časopis za ekonomsku teoriju i praksu, 22(1), pp. 7-19.

Duan, Q. (2017). A study of the influence of learning organization on organizational creativity and organizational communication in high tech technology. Eurasia Journal of Mathematics, Science and Technology Education, 13(6), pp. 1817-1830. https://doi.org/10.12973/eurasia.2017.00699a

Duncan, J. (1973). Comunications theory and problems of knowledge flow in management. The Journal of Business Communication, 11(1), pp. 3-14.

Encinas Orozco, F. C. (2014). Orientación de las organizaciones públicas al aprendizaje organizacional. El caso de los organismos descentralizados en el Estado de Sonora, México. Estudios Gerenciales, 30(130), pp. 10-17. https://doi.org/10.1016/j.estger.2014.01.010

Fernández, R. C. (2007). La dirección estratégica en la sociedad del conocimiento. Parte I . El cuadro de mando integral como herramienta para la gestión. ACIMED, 15(6).

Huang, P. y Yao, C. (2017). Effect of learning organization on organizational communication and organizational creativity in high-Tech Industry. Eurasia Journal of Mathematics, Science and Technology Education, 13(12), pp. 7723-7730. https://doi.org/10.12973/ejmste/78702

López, M. del S., Cabrales, F. y Schamal, R. (2005). Gestión del conocimiento: una revisión teórica y su asociación con la universidad. Panorama Socioeconómico, (30).

Manzano, O., Peñaranda, M. y Páez, H. (2019). Impact of information and 
communication technologies on organizational learning. Journal of Physics: Conference Series, 1409(1). https://doi.org/10.1088/17426596/1409/1/012019

Martín, I. (2007). Retos de la comunicación corporativa en la sociedad del conocimiento: de la gestión de información a la creación de conocimiento organizacional. Signo y Pensamiento, 26(51), pp. 52-67.

Martínez Crespo, J., y Giraldo Marín, L. M. (2012). La organización y su adaptación a las tecnologías de la información y la comunicación en procesos de gestión del conocimiento. Semestre Económico, 15(32), pp. 161-184.

Marulanda, C. E., Giraldo, J. A. y López, M. (2013). Evaluación de la Gestión del Conocimiento en las Organizaciones de la Red de Tecnologías de Información y Comunicaciones del Eje Cafetero en Colombia. Información Tecnológica, 24(4), pp. 105-116. https://doi.org/10.4067/S0718-07642013000400012

Michailova, S. y Sidorova, E. (2011). From group-based work to organisational learning: the role of communication forms and knowledge sharing. Knowledge Management Research y Practice, 9(1), pp. 73-83. https://doi. org/Doi 10.1057/Kmrp.2011.4

Nonaka, I., y Takeuchi, H. (2000). La empresa creadora de conocimiento. Gestión Del Conocimiento, pp. 1-9.

Núñez Paula, C. I. (2004). La gestión de la información, el conocimiento, la inteligencia y el aprendizaje organizacional desde una perspectiva socio-psicológica. Acimed, 12(3), pp. 1-75. http://scielo.sld.cu/scielo.php?script=sci_ arttext\&pid=S1024-94352004000300004\&lng=es\&nrm=iso\&tlng=es

Oviedo-García, M. Á., Castellanos-Verdugo, M., Riquelme-Miranda, A. y García del Junco, J. (2014). La relación entre aprendizaje organizacional y los resultados en la Administración Pública. Revista Europea de Dirección y Economía de La Empresa, 23(1), pp. 1-10. https://doi.org/10.1016/j.redee.2013.02.001

Senge, P. (1990). The fifth discipline. The art and practice of the learning organization. New York, EEUU: Currency Doubleday.

Senge, P., Kleiner, A., Roberts, C., Ross, R., Roth, G. y Smith, B. (2000). La danza del cambio. Los retos de sostener el impulso en organizaciones abiertas al aprendizaje. Bogotá, Colombia: Editorial Norma.

Tobio, M. (1997). El papel de la comunicación interna en las organizaciones actuales. Anuario de Comunicación, pp. 184-185. 